\title{
On Digital Image Representation by the Delaunay Triangulation
}

\author{
Josef Kohout \\ Department of Computer Science and Engineering, University of West Bohemia, \\ Univerzitní 22, 30614 Plzeň, Czech Republic \\ besoftakiv.zcu.cz
}

\begin{abstract}
This paper deals with a transformation of raster grey-scale images into a geometric representation by the Delaunay triangulation. It discusses the influence of image filtering techniques and methods for the evaluation of significance of pixels on the conversion process. Furthermore, it proposes several novel approaches for a compression of the Delaunay triangulation and compares them with existing solutions.
\end{abstract}

Keywords: image coding, Delaunay triangulation, compression.

\section{Introduction}

A digital image is nowadays usually represented by a rectangular grid of pixels, where each pixel contains a colour value or a greyscale luminance. In order to minimize the storage costs, the grid is very often transferred and stored in a compact form such as well-known GIF, PNG and JPEG. This representation suffers from several disadvantages. First, it cannot be easily processed in its compact form. Next, scaling and rotation operations typically introduce some distortion to the image.

Therefore, many researchers have focused recently on alternative geometric representations, such as triangular meshes. Geometric representations are applicable since the pixels of an image can be considered 3D points in a space in which $\mathrm{x}$ and $\mathrm{y}$ coordinates are the rows and columns of the image, and z-coordinate is either the grey level or colour value. As it would not be very useful to represent an image with $N$ pixels by a triangulation with the same number of vertices, a triangulation such that it has fewer vertices but it still sufficiently approximates the original mesh is very often needed to be found. From this triangulation, the corresponding image can be easily reconstructed by the interpolation among vertices of the mesh. Let us note that, if bilinear interpolation is exploited, this can be done in real time (especially, if graphics adapters are exploited).

Existing methods for the conversion of digital images from the traditional raster representation into a geometric representation can be subdivided into three main categories according to the goal they want to achieve as follows. First, there are methods that produce geometric representations that enhance the quality of further image processing. The representations are not compact as they contain usually as many vertices as the raster. Majority of these methods creates the data dependent 
triangulation (DDT) where triangle edges match the edges in the image and they differ only in cost functions used to detect an edge and optimisations [1], [16], [17].

In the second category, there are methods that produce compact (i.e., only a subset of vertices is kept) but highly imprecise representations. They find its use in applications of non-photorealistic rendering where details are unwanted because they make an understanding of the information presented by the image more difficult. A typical application of such representations is described in [10]. From existing methods that belong to this category, let us describe two interesting.

Prasad et al. [13] proposed a technique that starts with the detection of edges in the input image using the Canny operator. The detected edges are used as constraints for the constrained Delaunay triangulation that is afterwards computed. For every constructed triangle one colour computed as the average of colours of pixels covered by the triangle is assigned. Adjacent triangles with similar colours are merged together forming a polygon for which a common colour is chosen. The process results in the polygonal representation of the input image.

Kreylos et al. [11] describes an interesting approach that starts with the Delaunay triangulation of a randomly chosen subset of vertices that is successively improved by choosing different vertices governed by a simulated annealing algorithm. A drawback of their approach is that the final triangulation contains a lot of long and narrow triangles that may be difficult to efficiently encode. The approach was later exploited by Cooper et al. [3] for the surface reconstruction from a set of images. Instead of picking a random subset for the initial approximation, they, however, choose detected important points (typically, corners and edges).

The last category consists of methods that attempt to balance the compactness and the quality of the produced geometric representations that, if efficiently encoded, are suitable for the storing of digital photos. These representations are very often adaptive triangulations that differ in the way how they were obtained. In general, we can identify two basic strategies how to create an adaptive triangulation. The first one generates an adaptive triangular mesh by starting with two triangles covering the whole image area and then successively splitting them in order to reduce the approximation error. Alternatively, the algorithm can start with a fine mesh and successively make it coarser until the approximation error is above the desired tolerance. The question is which triangle should be split or which vertex should be removed in the next step and that it is not a simple task is demonstrated by two straightforward approaches described in [9] and [2] that either do not preserve well sharp edges in images or produce meshes with many vertices. Let us describe some more sophisticated approaches.

Starting with two initial triangles and their corresponding approximated image, Rila et al [14] successively construct the Delaunay triangulation as follows. A vertex, in which the approximation is the poorest, is inserted into the triangulation, which results in the construction of new triangles. These triangles are interpolated, i.e., a new approximation is obtained, and the next point to be inserted is found. The process stops when the required quality of the approximation is reached. The authors also describe a technique for the storing of the created mesh. As the Delaunay triangulation of a set of points is unique, it is necessary to store just vertex positions and their grey levels. An array of $N$ bits such that it contains 1 at positions appropriate to the vertices of the constructed triangulation and 0 elsewhere is constructed and 
compressed using a RLE (Run Length Encoding) approach. The grey levels are encoded using a fixed-length uniform quantizer of 5 bits.

García et al [8] choose a predefined number of pixels from image by applying a non-iterative adaptive sampling technique, which detects pixels on edges present in the image, and triangulate the corresponding points of these pixels using the Delaunay triangulation. Afterwards, triangles are further subdivided as long as the error of the approximation does not drop below some threshold. Although the authors were able to achieve better results (in the compression ratio as well as in the quality of the representation) than the authors of straightforward approaches, the results are, in our opinion, still far from being perfect.

In the approach described by Galic et al [7], a vertex with the poorest approximation is found using the same criteria as Rila et al. [14] in every step of their algorithm and the triangle containing this vertex is split into two new triangles by the height on its hypotenuse. The centre of the hypotenuse becomes an additional vertex of the representation. The advantage of this hierarchical splitting process is that it forms a binary tree structure that can be efficiently stored using just one bit per node. For the encoding of grey levels, the authors use the Huffman compression. In their paper, they also discussed various interpolation techniques and finally they decided to use edge-enhancing diffusion interpolation for their experiments instead of commonly used piecewise linear interpolation.

More recently, Demaret et al. [4] proposed an algorithm that computes the Delaunay triangulation of all vertices and after that it successively decimates this triangulation by removing the least significant vertex in every step. A vertex is considered to be the least significant, if its removal leads to the approximation of the original image with the smallest mean square error (MSE). The authors were able to achieve the compression ratio comparable with JPEG and the same or, especially, for higher compression ratios, even better quality of the image representation. On the other hand the proposed algorithm consumes a lot of time.

In our research, we followed the approach by Demaret et al. and investigated various problems related to the transformation of the traditional pixel representation into the representation by the Delaunay triangulation and to the encoding of the produced triangulation. The following text is structured into five sections. The next section gives an overview of the transformation process. In Section 3, several image filtering techniques and their impact on the quality of the final representation is discussed. Section 4 deals with the construction of the Delaunay triangulation itself and compare various cost functions. The encoding of the created triangulation is discussed in Section 5 and the paper is concluded in Section 6.

\section{Overview of the Transformation Process}

Fig. 1 gives a schematic overview of the complete transformation from the raster representation into a geometric representation stored in a compact form in a bitstream and the overview of the corresponding reverse transformation from this form into a raster of pixels. Let us note that we deal, similarly to many other authors, with greyscale images only because every component of colour images can be considered as a grey-scale image and can be processed individually. 


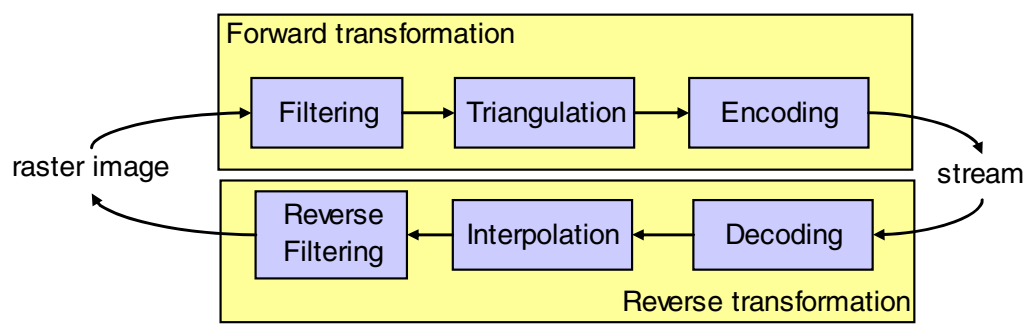

Fig. 1. A schematic view on transformation processes

The first step of the forward transformation is a lossless filtering of the input image. The goal of this step is to modify the image in such a way that it may be represented in the requested quality by a triangulation with a lower number of vertices, i.e., by a more compact triangulation. The details about filtering and its impact are given in the next section.

Each pixel of the filtered image stands for one vertex with coordinates $\mathrm{x}$ and $\mathrm{y}$ that denote the position of this pixel in the image and the coordinate $\mathrm{z}$ that is its grey level. The Delaunay triangulation of these vertices is constructed. For readers not familiar with the Delaunay triangulation, let us define the Delaunay triangulation as a triangulation such that the circum-circle of any triangle does not contain any of the given vertices in its interior (also called the empty circum-circle criterion). Let us note that the Delaunay triangulation can be computed in $O(N)$ expected time where $N$ is the number of vertices.

We decided to use the Delaunay triangulation mainly because of its two important properties. First, the Delaunay triangulation maximizes the minimal angle and, therefore, it contains the most equiangular triangles of all triangulations (i.e., it limits the number of too narrow triangles that may cause problems during the interpolation). Next, if no four points lie on a common circle, then the Delaunay triangulation is unique, i.e., it is sufficient to store vertices only and the same triangulation can be later recalculated. It is clear that this condition is violated for vertices obtained directly from pixels. This problem can be, however, easily solved by a random subpixel perturbation of vertices.

An initial significance of vertices is evaluated and all vertices are put into a priority queue ordered by this significance. We use the heap as an efficient implementation of the priority queue because it ensures $\log (N)$ for every operation. After that the iterative process starts. In every step, the least significant vertex is taken from the queue and removed from the triangulation, which may lead to a reevaluation of the significance of some of the remaining vertices. The process stops when the requested quality of the representation is achieved. The details concerning the evaluation of the significance of vertices and the removal of vertices are described in Section 4.

After the final Delaunay triangulation is obtained, it may be compressed and stored in a compact form onto a disk, from which it may be later, in the reverse transformation process, decompressed. The techniques suitable for the compression of the Delaunay triangulation are discussed in Section 5.

In the reverse transformation, each triangle of the Delaunay triangulation is rasterized, i.e., converted into a set of pixels, and shaded (we use the bilinear 
interpolation of grey values stored in the vertices of this triangle to get values for the created pixels). The result of this interpolation process is a raster image that is further transformed by the application of reverse filter into the final reconstructed image.

\section{Image Filtering}

Filtering transforms the image with the goal of improving the compression ratio whilst keeping the requested quality intact. While filtering techniques are thoroughly exploited in various raster compact formats (particularly in PNG), as far as we know, there is no paper considering their use for the transformation of the raster representation into a geometric one. In this section, we, therefore, discuss the importance of filtering techniques in the transformation process and their impact on the quality of the final representation.

For an easier understanding of the problem, let us resort to one dimensional case. Fig. 2 shows a function that should be approximated by a piecewise linear function with the allowed approximation error $\varepsilon$. In its original form, an approximation that connects the ending points of the given function, $p_{a}$ and $p_{b}$, is not possible because its error is out of the specified tolerance. It is, therefore, necessary to introduce the third vertex, $p_{c}$, into the approximation to fulfil the criterion. If the input function is, however, filtered using a simple SUB filter (will be described later), the approximation by $p_{a}$ and $p_{b}$ is possible. It means that by the filtering we reduced the number of vertices from three to two, i.e., we improved the compression ratio.

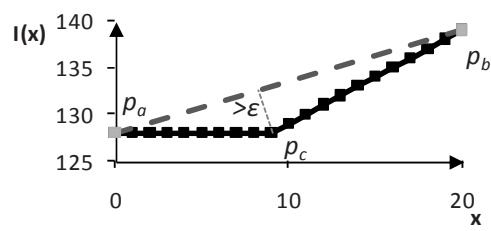

a) the original function

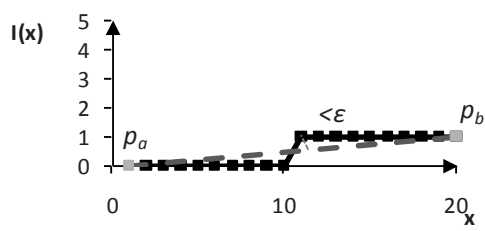

b) the filtered function

Fig. 2. An edge approximation (dashed line) of the original and filtered function (solid line)

We tried three image filters used in PNG. The first one is already mentioned SUB filter that computes differences between neighbouring pixels using the formula:

$$
\operatorname{SUB}(x)=\operatorname{IMG}(x)-\operatorname{IMG}(x-1),
$$

where $x$ ranges from zero to the number of pixels in the image minus one and IMG() refers to the grey value of the pixel in the image corresponding to the specified position. Unsigned arithmetic modulo 256 is used, so the outputs fit into bytes (e.g., $1-2=255)$. For all $x<0$, we assume $\operatorname{IMG}(x)=0$. In order to reverse the effect of the SUB filter after the interpolation, the output is computed as:

$$
\operatorname{IMG}(x)=\operatorname{SUB}(x)+\operatorname{IMG}(x-1) .
$$


The AVG filter transmits the difference between the value of a pixel and the average of the two neighbouring pixels (left and above) used as a prediction of this value. The formulas for forward and reverse filter can be written as:

$$
\begin{aligned}
\operatorname{AVG}(x) & =\operatorname{IMG}(x)-((\operatorname{IMG}(x-1)+\operatorname{IMG}(x-c x)) / 2, \\
\operatorname{IMG}(x) & =\operatorname{AVG}(x)+((\operatorname{IMG}(x-1)+\operatorname{IMG}(x-c x)) / 2,
\end{aligned}
$$

where $c x$ denotes the horizontal size of the image.

As the previous filter, the PAETH filter also transmits the difference between the real value and the predicted value of a pixel, however, the prediction is calculated from the three neighbouring pixels (left, above, upper left) by the algorithm developed by Alan W. Paeth (see http://www.w3.org/TR/PNG/).

Despite our expectations, the experiments proved that these filtering techniques are not useful; we obtained even worse results with them than without. Fig. 3 shows images of fruits that were reconstructed from triangulations with $93.4 \%$ of the original amount of vertices (i.e., only an insignificant amount of vertices was removed) when filtering techniques were applied. Artefacts are clearly visible.

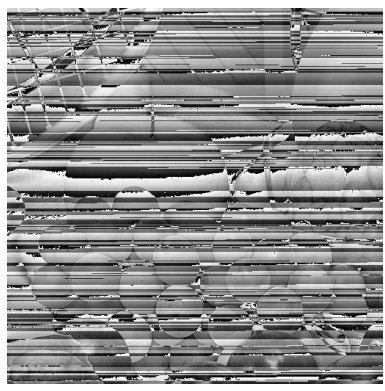

a) SUB

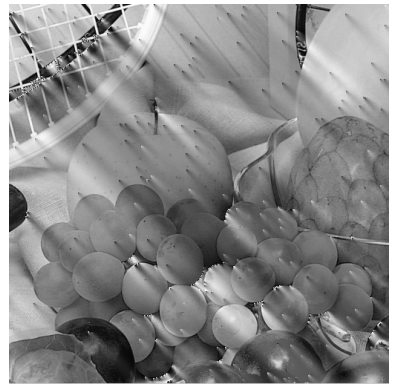

b) AVG

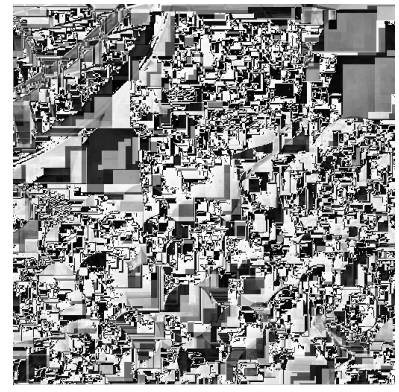

c) PAETH

Fig. 3. The artefacts caused by various filtering techniques

We identified several reasons for such behaviour. The most important fact is that by filtering we introduce a dependency between pixels and, therefore, if one pixel is reconstructed with an error, this error is distributed over the rest of pixels, which may cause unexpected artefacts. Let us consider the following example. The SUB filter transforms pixels $[0,0,10,10,10]$ into filtered values $[0,0,10,0,0]$. If $2^{\text {nd }}$ value is not stored and has to be reconstructed, we get values $[0, \mathbf{5}, 10,0,0]$. The reverse SUB filter propagates the error and gives pixels $[0, \mathbf{5}, \mathbf{1 5}, \mathbf{1 5}, \mathbf{1 5}]$.

The problem is also that although the filtering flattens the image, it does not create sufficiently large places with constant value but, on the contrary, it introduces a lot of edges into the image. It makes the approximation process uneasy as it leads to a rapid degradation of quality.

Having a bad experience with the filtering, we cease to use it in our next experiments. Nevertheless, we believe that the idea of filtering of image in the 
preprocessing is not bad in general but one has to come with the filtering technique where the filtered values are more independent and thus less liable to errors.

\section{Delaunay Triangulation}

After the pixels are transformed into vertices, the Delaunay triangulation of these vertices is computed and this triangulation is successively decimated by the deletion of the least significant vertex from the triangulation in every step. The deletion consists of two steps. First, a fan of triangles sharing the vertex to be deleted is removed from the triangulation, which results in a star-shaped hole. Next, the hole is filled by new triangles fulfilling the Delaunay criterion - see Fig. 4.

For the triangulation of the hole, we use the approach by Devillers [5] because of its simplicity and efficiency (it requires $O(m \cdot \log m)$, where $m$ is the number of vertices forming the hole). It works as follows. For each triple of topologically consecutive vertices $q_{\mathrm{i}}, q_{\mathrm{i}+1}, q_{\mathrm{i}+2}$ along the boundary of the hole, i.e., for a candidate for the triangle, a weight computed as a function of coordinates $q_{\mathrm{i}}, q_{\mathrm{i}+1}, q_{\mathrm{i}+2}$ and $p$ is assigned. All candidates are put into a priority queue ordered by their weights. After that an iterative filling process starts. In every step of this process, a candidate at the head of the queue is taken and the corresponding triangle is constructed. The candidates $q_{\mathrm{i}-1}, q_{\mathrm{i}}, q_{\mathrm{i}+1}$ and $q_{\mathrm{i}+1}, q_{\mathrm{i}+2}, q_{\mathrm{i}+3}$ that overlap the newly constructed triangle are changed to $q_{\mathrm{i}-1}, q_{\mathrm{i}}, q_{\mathrm{i}+2}$ and $q_{\mathrm{i}}, q_{\mathrm{i}+2}, q_{\mathrm{i}+3}$ and their weights are recalculated. The process stops when the hole is filled.

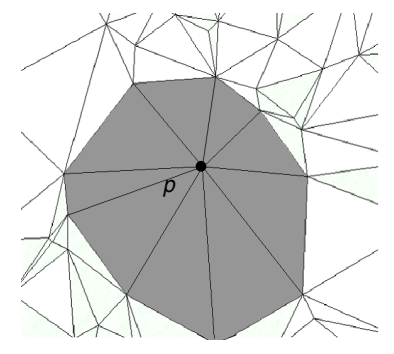

a) triangles to be removed

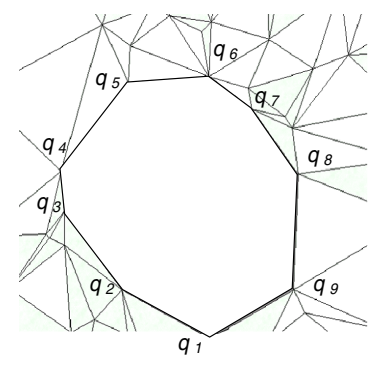

b) the hole

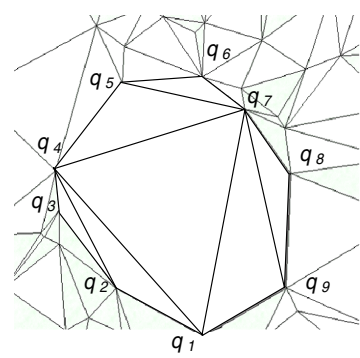

c) Delaunay retriangulation

Fig. 4. The deletion of the point $p$ from the Delaunay triangulation

As for the evaluation of the significance of vertices, we proposed and tested several methods. The simplest one, denoted as RND, assigns a random significance to vertices at the beginning of the decimation process and does not modify it during the process. Thus it means that vertices are removed from the triangulation in a random fashion. A more sophisticated method, called MARR, computes the significance of vertices as the results of Marr-Hildreth edge detection operator [12], i.e., vertices that form edges in the image are more significant. As in the RND method, the significance is not recalculated during the decimation. 
Other three methods start with the computation of significance of a vertex $p$ as the negative absolute difference of the grey value held by this vertex and the value computed as the distance weighted average of grey values of its neighbouring vertices $q$, i.e., vertices connected with $p$ by an edge:

$$
\left|\operatorname{grey}(p)-\frac{\sum_{q} \operatorname{grey}(q) \cdot\|p-q\|}{\sum_{q}\|p-q\|}\right| .
$$

When a vertex is deleted from the triangulation, the significance of all vertices that formed the hole, i.e., they were originally connected by an edge with the deleted vertex, is recalculated. While the first method, which is denoted as DISTW, performs the recalculation using the same formula, the second method called ERRDIST updates the significance in a way similar to the Floyd-Steinberg error diffusion technique [6]. It modifies the significance of a vertex by adding a fraction of the significance of deleted vertex that is appropriate to the distance between these two vertices. The third method called TRIMSE updates the significance as follows. For each newly constructed triangle, it first computes its mean square error (MSE), i.e., it computes the MSE between the grey values of pixels covered by this triangle in the original image and the corresponding values obtained by the bilinear interpolation of grey values of triangle vertices. The significance of a vertex is then recalculated as the sum of MSE of triangles that share this vertex. Let us note that this method is, indeed, slower than the previous two methods.

The last method, denoted as BRUTE, is based on a brute-force idea to calculate the significance of a vertex as the sum of MSE of triangles that would be constructed if this vertex was deleted. To speed up the processing, the initial significance of a vertex is computed simply as the negative square difference of the grey value held by this vertex and the average of grey values held by two its neighbouring vertices (left and right). When a vertex is deleted, the significance of all vertices that formed the hole is recalculated using the described brute-force idea. It is without any doubt that this method is the most time consuming but we may expect the best results.

Fig. 5 shows images of Lena reconstructed from Delaunay triangulations with 10000 vertices (i.e., $96 \%$ of vertices was removed) computed using different methods for the evaluation of vertex significance. It is not surprising that methods that take the MSE of triangles into an account give better results. On the other hand the MARR method provides us unexpectedly with results of a low quality. The reason is that there is not a sufficient amount of vertices to represent also areas with a smooth change of intensity (e.g., in face) in a good quality because too many vertices were wasted to represent areas with sharp edges in an outstanding quality (see the feather).

For each of proposed methods of the vertex significance evaluation, we investigated the degradation of the quality of the geometric representation in the dependency on the amount of removed vertices for a set of grey scale images. The results for three popular images from this set are presented in Fig. 6. Let us note that similar results were obtained also for the remaining tested images. As it can be seen from graphs, the quality of the representation degrades quite quickly until the algorithm removes approximately $25 \%$ of vertices, after that the quality decreases almost linearly in a slow pace until another threshold of about $90 \%$ removed vertices 
is reached. From that moment, the quality rapidly drops down. An interesting observation is that in this last period, all methods (including the RND method based on a random selection of vertices to be removed) produce similar results. It means that an application that calls for a triangulation with a few vertices only (e.g., in nonphotorealistic rendering), does not need to pay much attention which method for the evaluation of vertex significance to use.

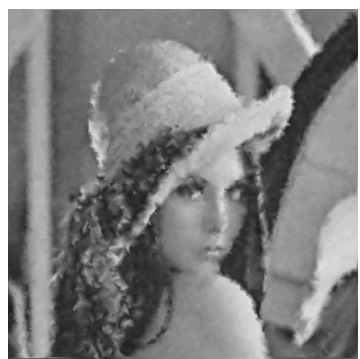

a) $\mathrm{RND}, \mathrm{PSNR}=24.26$

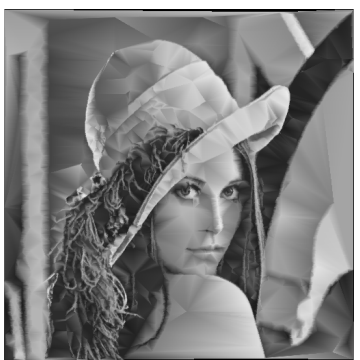

d) ERRDIST, PSNR=22.33

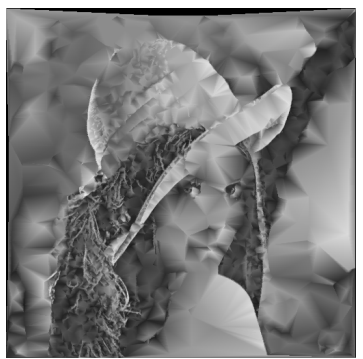

b) MARR, PSNR $=17.91$

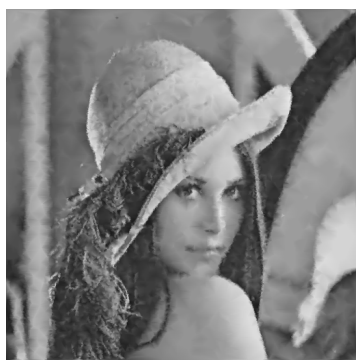

e) TRIMSE, PSNR=27.14

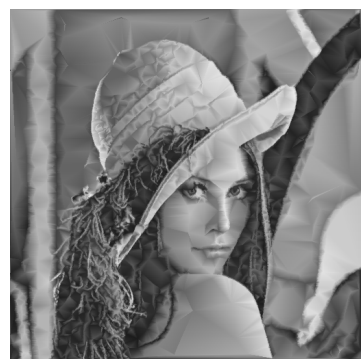

c) DISTW, PSNR $=26.84$

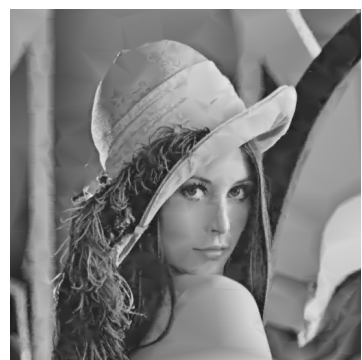

f) BRUTE, PSNR $=30.78$

Fig. 5. The comparison of Lena images reconstructed from triangulations with 10000 vertices computed using different methods

As it can be further seen, the curve of quality achieved by the slowest BRUTE method is the upper bound for all other curves, while the curve achieved by the simplest (and fastest as well) RND method stands for the lower bound. All methods except for these two methods produce very similar results despite that they use different evaluation heuristics. This introduces a question whether it makes sense to try to develop a sophisticated evaluation method because it is likely that such a method would be time consuming but would not bring a significant improvement.

\section{Encoding}

If we cease to retain the connectivity of triangles and store the geometry only (the Delaunay triangulation can be recomputed from the geometry), 5 bytes are required per one vertex $(2+2$ for the $\mathrm{x}$ and $\mathrm{y}$-coordinates, 1 for the grey value). Even if it 
contains a few vertices only, the triangulation in this raw form consumes a lot of bytes and, therefore, it is not suitable for storing. A more compact form is necessary. We experimented with several compression methods and, in this section, we present results of our experiments.
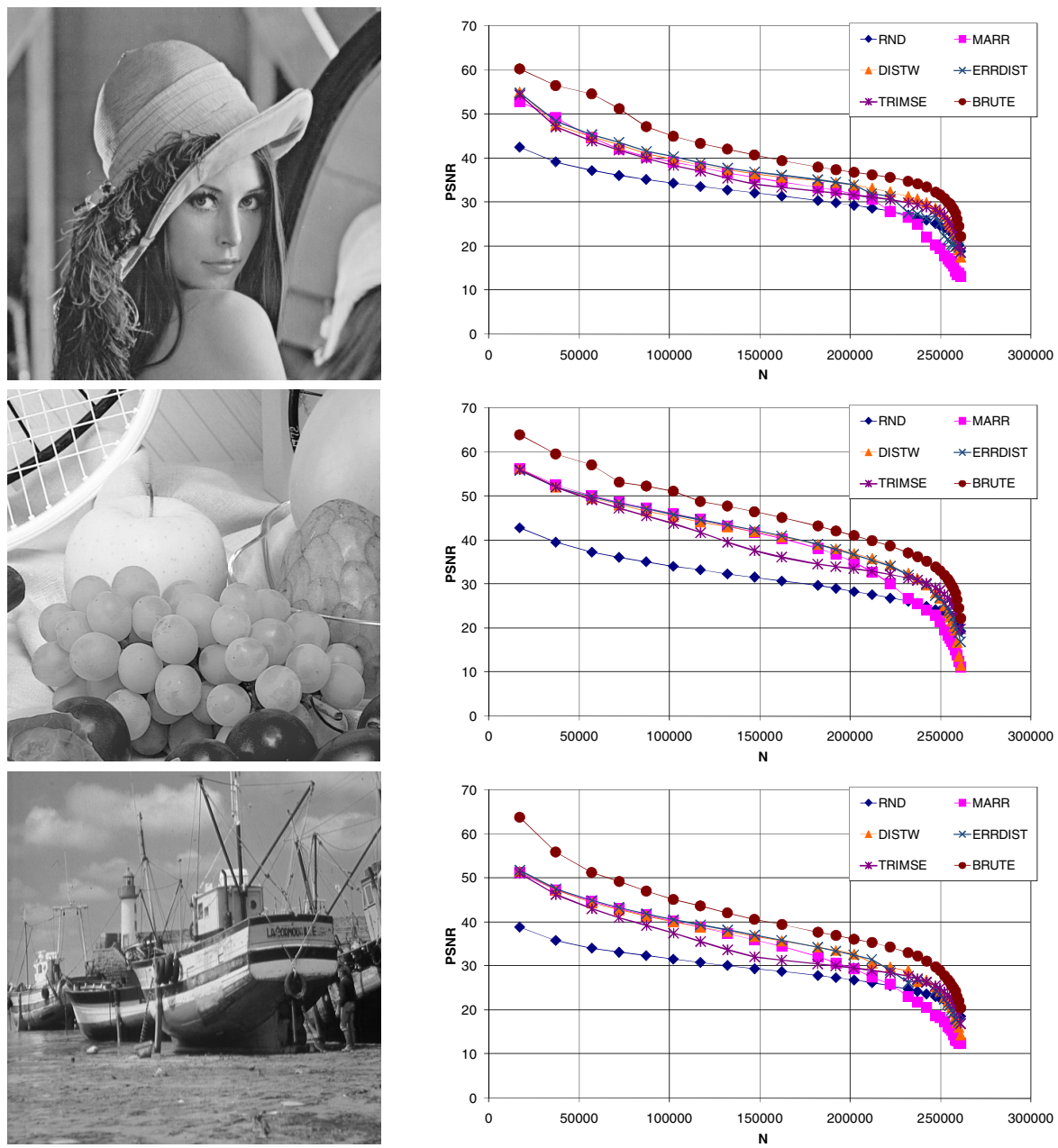

Fig. 6. The dependency of quality (measured in PSNR) on the number of removed vertices for three popular 512x512 grey-scale images

Edgebreaker proposed by Rosignac [15] is probably the most often used algorithm for a compression of an arbitrary triangulation. Edgebreaker visits triangles in a spiralling order and generates a string of symbols from the set $\{\mathrm{C}, \mathrm{L}, \mathrm{E}, \mathrm{R}, \mathrm{S}\}$. This string describes the connectivity, i.e., it indicates how the mesh can be rebuilt. Using the Huffman compressor, it can be efficiently encoded so that two bits per triangle are guaranteed. The geometry, i.e., coordinates and grey levels of vertices are encoded as 
follows. When a triangle, say $p_{\mathrm{a}}, p_{\mathrm{b}}, p_{\mathrm{c}}$, is visited and the far vertex $p$ of its adjacent triangle has not been processed yet, a prediction $q$ is computed using the parallelogram predictor and the algorithm stores the differences between this predictor and the vertex $p-$ see Fig. 7. The number of bits used to store these differences depends on how accurate the predictions of vertices are. Theoretically, if the predictor were able to give always an accurate prediction (i.e., $q$ and $p$ are the same), it would be possible to avoid the storing of geometry. In practice, however, this case does not exist.

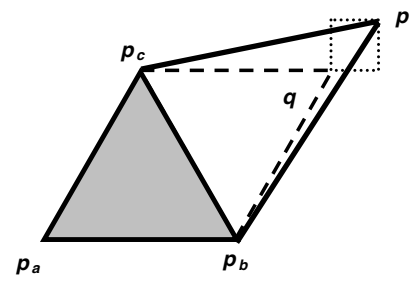

Fig. 7. The prediction $q$ of the vertex $p$

An advantage of this algorithm is that it can compress any triangulation, not only the Delaunay triangulation, and, if the parallelogram predictor is used, it runs incredibly fast. On the other hand, its implementation for triangulations with boundaries is not as simple as for closed meshes. Another drawback is that the connectivity has to be stored even for the Delaunay triangulation because it is needed for decoding of coordinates.

Therefore, we propose several simple methods that encode the geometry only. The first one, VXPATH, successively visits all vertices storing the differences between the currently inspected vertex and the previously visited vertex. Let us note that the differences in $\mathrm{x}$ and $\mathrm{y}$-coordinates are stored separately from the differences in grey levels (i.e., in z-coordinates). Vertices are visited in such an order that the differences in $\mathrm{x}$ and $\mathrm{y}$-coordinates are minimized. Being in a vertex $p$, the algorithm thus continues with the vertex $q$ such that it has not been already visited and the value:

$$
\left|p_{x}-q_{x}\right|+\left|p_{y}-q_{y}\right|
$$

is minimal. An advantage of this approach is that it does not require the connectivity for the decoding process and it offers a compactness as the differences should be very small. On the other hand, this brute-force algorithm runs in $O\left(N^{2}\right)$, which means that it takes a lot of time (especially, if the triangulation contains a large number of vertices).

The second method, called TRPATH, enhances the currently described method in two things. First, the vertices are processed in a different order (but by the same way) as follows. The algorithm traverses the triangulation in the depth-first order (similarly to Edgebreaker) and whenever a new triangle is visited, its still not processed vertices are processed. As vertices are handled in linear time, the time consumption is significantly reduced. The second improvement is that the TRPATH does not use a fixed number of bits for all differences, which makes the algorithm slightly more complex but promises lower storage costs. 
The last method, which is called KORILA, is based on the idea presented by Rila et al. [14] to store the geometry as a bitmap that contains 1 at positions corresponding to the vertices of triangulation and 0 elsewhere. This bitmap is compressed independently by a Run Length Encoding (RLE) and by the Huffman encoding and the result that is smaller is stored into the output file. Let us note that Huffman has proved to outpace RLE in all our experiments. Grey-values and values obtained by the application of the SUB filter on grey levels (see Section 3) are also independently compressed using the Huffman approach and the smaller outcome is stored. In our experiments, the filtering has proved to be useful especially for larger meshes where intensities do not change as rapidly as in small triangulations.

Table 1 brings the results obtained for triangulations with 50000 vertices computed using the BRUTE method for the three popular images. While VXPATH and TRPATH methods achieved a similar compression ratio for all three images, other two methods show a different behaviour. The reason of this for EdgeBreaker is that the triangulation may be very irregular (particularly for images with a large amount of sharp edges) as it can be seen in Fig. 8 and, therefore, the predictor used in Edgebreaker often produces highly inaccurate predictions, which leads to lower compression ratio. The KORILA method is sensitive to input data as the Huffman encoding achieves worse results for images that contain a large scale of grey levels.

Table 1. The comparison of sizes of output files produced by various compression schemes for triangulations with 50000 vertices of three popular $512 \times 512$ grey-scale images

\begin{tabular}{lrrrrr}
\hline Image & PSNR & Edgebreaker & VXPATH & TRPATH & KORILA \\
\hline Lena & 36.29 & 152624 & 175012 & 138450 & 50804 \\
Fruits & 39.95 & 171464 & 162512 & 156106 & 70769 \\
Boat & 35.30 & 273648 & 175012 & 144288 & 69307 \\
\hline
\end{tabular}
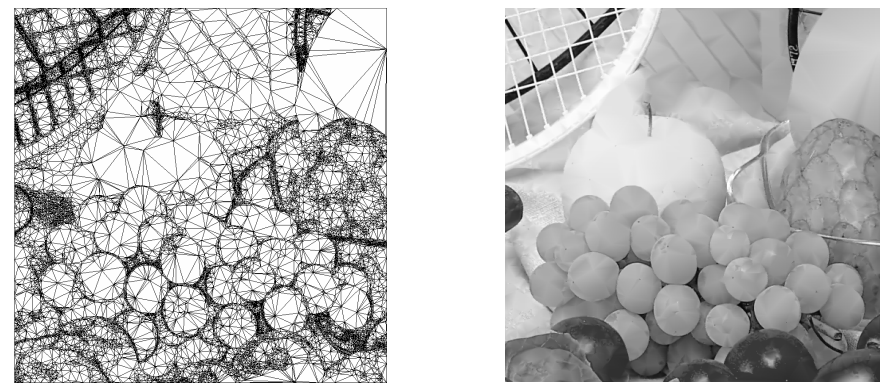

Fig. 8. The Delaunay triangulation with 15000 vertices and the corresponding reconstructed image $(\mathrm{PSNR}=33.90)$

We also tried to zip the output files in order to achieve a better compression ratio. The results are shown in Table 2. While Edgebreaker and VXPATH produce files that are highly compressible, the KORILA method gives outputs not compressible at all. 
Table 2. The comparison of sizes of zipped output files produced by various compression schemes for triangulations with 50000 vertices of three popular 512x512 grey-scale images

\begin{tabular}{lrrrrr}
\hline Image & PSNR & Edgebreaker & VXPATH & TRPATH & KORILA \\
\hline Lena & 36.29 & 66566 & 52470 & 90602 & 50260 \\
Fruits & 39.95 & 79620 & 57135 & 100998 & 70751 \\
Boat & 35.30 & 110419 & 53677 & 115788 & 69377 \\
\hline
\end{tabular}

The dependency of file sizes of outputs produced by the KORILA method on the number of vertices in triangulations is presented in Table 3. Let us note that the filtering of grey values in prior to encoding proved to be useless for the smallest three triangulations. We compared the achieved results also with JPEG and JPEG2000. As it can be seen from Table 4, both compression techniques outpace the KORILA method; JPEG2000 even produces files of half sizes.

Table 3. The sizes of output files produced by the KORILA method for triangulations with 50000 down to 1000 vertices

\begin{tabular}{llrrrrrrr}
\hline & & 50000 & 30000 & 20000 & 10000 & 5000 & 2000 & 1000 \\
\hline Lena & Size & 50804 & 33057 & 23877 & 14331 & 9379 & 6262 & 5211 \\
& PSNR & 36.29 & 34.78 & 33.41 & 31.00 & 28.37 & 24.77 & 21.37 \\
Fruits & Size & 70769 & 45521 & 32332 & 18692 & 11648 & 7324 & 5862 \\
& PSNR & 39.95 & 37.18 & 35.27 & 32.00 & 28.92 & 24.52 & 22.37 \\
Boat & Size & 69307 & 44930 & 32066 & 18620 & 11622 & 7293 & 5838 \\
& PSNR & 35.30 & 33.10 & 31.00 & 27.83 & 25.11 & 22.46 & 20.55 \\
\hline
\end{tabular}

Table 4. The sizes of output files produced by JPEG and JPEG2000

\begin{tabular}{llrrrrr}
\hline & \multicolumn{3}{c}{ JPEG } & & \multicolumn{3}{c}{ JPEG2000 } \\
\hline Lena & Size & 38467 & 16159 & 3147 & 25538 & 11948 \\
& PSNR & 36.22 & 33.46 & 24.73 & 36.34 & 33.37 \\
Fruits & Size & 45584 & 21367 & 2677 & 33081 & 15755 \\
& PSNR & 39.31 & 35.28 & 24.58 & 39.97 & 35.31 \\
Boat & Size & 39357 & 16295 & 3024 & 26378 & 12325 \\
& PSNR & 35.36 & 31.00 & 23.03 & 35.26 & 30.95 \\
\hline
\end{tabular}

From results presented in this section, it is quite clear that the compression of computed triangulation is a very important issue. Even a small change of an existing method could dramatically change its typical compression ratio. Future research, therefore, should be more focused on the development of encoding techniques.

\section{Conclusion}

In this paper, we investigated a representation of digital image by the Delaunay triangulation computed from the original raster of pixels by successive removal of insignificant pixels (vertices). While a filtering of input image (by filters used in 
PNG), which was supposed to help to get triangulations with fewer vertices, proved to be useless, it may play a key role in the lossless compression of the computed triangulation. The results of performed experiments also lead us to the conclusion that attempts to develop a sophisticated method for evaluation of significance of vertices are a waste of time because it is likely that such a method would be time consuming but would not bring a significant improvement. Future research should be rather oriented towards a development of efficient triangle interpolation techniques because, in our opinion, the commonly used bilinear interpolation does not provide a sufficient quality. For public use of geometric representations, it is also vital to develop a compression scheme that would outpace JPEG and JPEG2000.

Acknowledgments. This work was supported by the Grant Agency of the Academy of Sciences of the Czech Republic (GA AV ČR) - project No. KJB101470701. The author would also like to thank I. Kolingerová and V. Skala from the University of West Bohemia for providing conditions in which this work has been possible.

\section{References}

1. Battiato, S., Gallo, G., Messina, G.: SVG rendering of real Images using data dependent triangulation. In: Proceedings of SCCG 2004, pp. 185-192 (2004)

2. Ciampalini, A., Cignoni, P., Montani, C., Scopigno, R.: Multiresolution decimation based on global error. The Visual Computer 13, 228-246 (1997)

3. Cooper, O., Campbell, N., Gibson, D.: Automatic augmentation and meshing of sparse 3D scene structure. In: Proceedings of 7th IEEE Workshop on Applications of Computer Vision, Breckenridge, pp. 287-293. IEEE Computer Society Press, Los Alamitos (2005)

4. Demaret, L., Dyn, N., Floater, M.S., Iske, A.: Adaptive thinning for terrain modelling and image compression. Advances in Multiresolution for Geometric Modelling, 321-340 (2004)

5. Devillers, O.: On deletion in Delaunay triangulations. In: Proceedings of SCG 1999, pp. 181-188. ACM Press, New York (1999)

6. Floyd, R.W., Steinberg, L.: An adaptive algorithm for spatial grey scale. In: Proceedings of the Society of Information Display, pp. 75-77 (1976)

7. Galic, I., Weickert, J., Welk, M.: Towards PDE-based image compression. In: Paragios, N., Faugeras, O., Chan, T., Schnörr, C. (eds.) VLSM 2005. LNCS, vol. 3752, pp. 37-48. Springer, Heidelberg (2005)

8. García, M.A., Vintimilla, B.X., Sappa, A.D.: Efficient approximation of grey-scale images through bounded error triangular meshes. In: Proceedings of IEEE International Conference on Image Processing, Kobe, pp. 168-170. IEEE Computer Society, Los Alamitos (1999)

9. Gevers, T., Smeulders, A.W.: Combining region splitting and edge detection through guided Delaunay image subdivision. In: Proceedings of IEEE International Conference on Computer Vision and Pattern Recognition, pp. 1021-1026. IEEE Computer Society Press, Los Alamitos (1997)

10. Grundland, M., Gibbs, Ch., Dodgson, N.A.: Stylized rendering for multiresolution image representation. Proceedings of SPIE 5666, 280-292 (2005)

11. Kreylos, O., Hamann, B.: On simulated annealing and the construction of linear spline approximations for scattered data. IEEE Transactions on Visualization and Computer Graphics 7, 17-31 (2001) 
12. Marr, D., Hildreth, E.C.: Theory of edge detection. In: Proceedings of the Royal Society, London B, vol. 207, pp. 187-217 (1980)

13. Prasad, L., Skourikhine, A.N.: Vectorized image segmentation via trixel agglomeration. Pattern Recognition 39, 501-514 (2006)

14. Rila, L.: Image coding using irregular subsampling and Delaunay triangulation. In: Proceedings of SIBGRAPI, pp. 167-173 (1998)

15. Rosignac, J.: Edgebreaker: Connectivity compression for triangle meshes. IEEE Transactions of Visualization and Computer Graphics 5, 47-61 (1999)

16. Su, D., Willis, P.: Image interpolation by pixel level data-dependent triangulation. Computer Graphics Forum 23, 189-201 (2004)

17. Yu, X., Morse, B.S., Sederberg, T.W.: Image reconstruction using data-dependent triangulation. IEEE Computer Graphics and Applications 21, 62-68 (2001) 